\title{
Pancreatitis aguda en niños con enfermedades hematooncológicas: aspectos clínicos y tratamiento
}

\author{
Lénica A. Chávez-Aguilar ${ }^{1}$ y Rubén Peña-Vélez ${ }^{2,3 *}$ \\ ${ }^{1}$ Servicio de Hematología Pediátrica, Centro Médico Nacional 20 de Noviembre, Instituto de Seguridad y Servicios Sociales de los Trabajadores \\ del Estado; ${ }^{2}$ Departamento de Gastroenterología y Nutrición, Instituto Nacional de Pediatría; ${ }^{3}$ Facultad de Medicina, Universidad Nacional Autónoma
} de México. Ciudad de México, México

\begin{abstract}
Resumen
La pancreatitis aguda es una enfermedad inflamatoria del páncreas. Se observa con mayor frecuencia en niños bajo tratamiento por alguna enfermedad hematooncológica y se asocia principalmente con la administración de L-asparaginasa. Identificar esta complicación de forma temprana y establecer un plan terapéutico adecuado puede mejorar el pronóstico y reducir el riesgo de otras complicaciones. En este trabajo se realizó una revisión crítica de la literatura actual, con especial énfasis en los aspectos clínicos, el diagnóstico y el tratamiento de la pancreatitis aguda en niños con cáncer.
\end{abstract}

Palabras clave: Pancreatitis aguda. Leucemia linfoblástica aguda. L-asparaginasa.

\section{Acute pancreatitis in children with hemato-oncological diseases: clinical aspects and treatment}

\begin{abstract}
Acute pancreatitis is an inflammatory disease of the pancreas. It is currently seen more frequently in children undergoing treatment for a hemato-oncological disease and it is mainly associated with the administration of L-asparaginase. The early identification of this complication and the establishment of an appropriate therapeutic plan can improve its prognosis and reduce the risk of other complications. In this article, we make a critical review of the current literature, with special emphasis on the clinical aspects, diagnosis, and treatment of acute pancreatitis in children with cancer.
\end{abstract}

Key words: Acute pancreatitis. Acute lymphoblastic leukemia. L-asparaginase.

\section{Introducción}

Los niños y adolescentes que se encuentran en tratamiento por enfermedades hematooncológicas comparten características clínicas que pueden incrementar el riesgo de desarrollar pancreatitis aguda (PA). Entre estos factores se considera que el cáncer es una enfermedad sistémica con compromiso inmunitario secundario. Además, estos pacientes reciben tratamientos prolongados con fármacos quimioterapéuticos tóxicos y
Correspondencia:

*Rubén Peña-Vélez

E-mail: rubenpevelez@ hotmail.com
Fecha de recepción: 22-04-2020

Fecha de aceptación: 22-06-2020

DOI: 10.24875/BMHIM.20000110
Disponible en internet: 12-01-2021

Bol Med Hosp Infant Mex. 2021;78(2):95-101

www.bmhim.com

1665-1146/@ 2020 Hospital Infantil de México Federico Gómez. Publicado por Permanyer. Este es un artículo open access bajo la licencia CC BY-NC-ND (http://creativecommons.org/licenses/by-nc-nd/4.0/). 
múltiples antimicrobianos, y tienen un mayor número de hospitalizaciones y de intervenciones quirúrgicas'.

La PA es una enfermedad inflamatoria del páncreas. Para su diagnóstico se requiere la presencia de al menos dos de los siguientes:

- Dolor abdominal compatible con PA.

- Elevación sérica de la amilasa o de la lipasa de hasta tres veces el valor normal para la edad.

- Hallazgos por imagen sugestivos de PA.

Se presenta una revisión narrativa de la literatura médica. Durante marzo y abril de 2020 se realizó una búsqueda en las bases de datos MEDLINE, ResearchGate, Mendeley y Google Scholar con los términos "Acute pancreatitis in children", "Pancreatitis in leukemia", "Asparaginase-associated pancreatitis" y términos relacionados. Para secciones específicas se utilizaron operadores booleanos. Se dio prioridad a artículos originales, revisiones sistemáticas y metaanálisis publicados en los últimos 10 años.

\section{Epidemiología}

La incidencia de PA ha aumentado en los últimos años. Algunos estudios recientes señalan que ocurre $1 / 10,000$ casos por año en la población pediátrica general ${ }^{3}$. En los niños con leucemia linfoblástica aguda (LLA) se puede presentar hasta en el $8.3 \%$ de los casos $^{4}$, y en protocolos de tratamiento de LLA ocurre en el $2-18 \%$ de los niños que recibieron tratamiento con L-asparaginasa ${ }^{5}$.

Un estudio realizado en un hospital pediátrico de tercer nivel de atención informó que el $48.8 \%$ de los niños atendidos por PA presentaba antecedentes oncológicos (LLA y tumores sólidos); además, reportó que el $51 \%$ de estos pacientes había recibido un agente quimioterapéutico en las 2 semanas previas al diagnóstico ${ }^{6}$. Otro estudio retrospectivo de 40 años, que incluyó 7250 protocolos de autopsia, identificó 44 casos con alteraciones histopatológicas de PA, principalmente asociados con cáncer y lupus eritematoso sistémico. En este estudio también se informó que el mayor número de casos ocurrieron en la última década?

\section{Etiología}

En los niños y adolescentes, la PA puede ser secundaria a enfermedades sistémicas, trauma abdominal, enfermedades hepatobiliares, anomalías estructurales pancreáticas, infecciones 0 enfermedades metabólicas, genéticas 0 idiopáticas ${ }^{8-10}$. Actualmente, debido al incremento en la incidencia de las enfermedades hematooncológicas $^{11}$ y de la pancreatitis, ambas pueden coexistir sin que exista una participación directa del cáncer en la etiología de la pancreatitis.

En los niños con cáncer, la principal etiología de la PA son los fármacos ${ }^{1}$. En los pacientes con LLA tratados con L-asparaginasa se ha descrito una predisposición genética ${ }^{12}$. También se puede presentar después de un trasplante de células progenitoras hematopoyéticas $(\mathrm{TCPH})$ y en pacientes con hipercalcemia ${ }^{13} \mathrm{O}$ hipertrigliceridemia ${ }^{14}$.

\section{Fármacos}

La L-asparaginasa es la piedra angular en el tratamiento de la LLA y de algunos tipos de linfoma. Es una enzima de origen bacteriano con capacidad de transformar la L-asparagina en ácido aspártico. La depleción extracelular de este aminoácido inhibe la síntesis proteica en los linfoblastos, induciendo apoptosis ${ }^{15}$. Actualmente, este fármaco es una causa bien conocida de $\mathrm{PA}^{16}$. Existen tres formulaciones de L-asparaginasa disponibles comercialmente, cuyas características, vida media y depleción se describen en la tabla 1.

La PA suele ocurrir, en promedio, 12 días después de un tratamiento con L-asparaginasa Escherichia coli nativa 026 días después del tratamiento con L-asparaginasa pegilada ${ }^{17}$. Este comportamiento se ha observado con las tres formulaciones de L-asparaginasa, aunque la menos relacionada es la L-asparaginasa Erwinia chrysanthemi y la descrita con más frecuencia es la L-asparaginasa pegilada ${ }^{16,17}$. Los síntomas de PA suelen aparecer en las primeras semanas de tratamiento, y la enfermedad puede ser recurrente si se continúa el tratamiento con L-asparaginasa tras un primer episodio. Aunado a esto, se ha descrito que ocurre con mayor frecuencia en adolescentes que en niños pequeños ${ }^{15}$.

Los corticoesteroides son parte importante del tratamiento de la LLA y de los linfomas, ya que inducen la detención del ciclo celular y la apoptosis ${ }^{18}$. Un metaanálisis reportó una tendencia de mayor riesgo de PA con el uso de dexametasona en comparación con prednisona $^{19}$.

Otros fármacos involucrados en el desarrollo de PA en los niños con LLA se especifican en la tabla 2. Muchas asociaciones se han establecido a partir de reportes de casos y series clínicas, por lo que se requiere realizar estudios controlados para definir el papel de estos fármacos en la patogénesis de la PA en los niños con cáncer. 
Tabla 1. Características de las diferentes formulaciones de L-asparaginasa

\begin{tabular}{|c|c|c|c|}
\hline Tipo de L-asparaginasa & $\begin{array}{l}\text { Vida media } \\
\text { (días) }\end{array}$ & $\begin{array}{l}\text { Depleción } \\
\text { (días) }\end{array}$ & Observaciones \\
\hline $\begin{array}{l}\text { Asparaginasa pegilada de } \\
\text { E. coli }\end{array}$ & $5.7 \pm 3$ & $26-32$ & $\begin{array}{l}\text { Conjugada en forma covalente con unidades de } \\
\text { monometoxipolietilenglicol. } \\
\text { Tratamiento de segunda o tercera línea en casos de hipersensibilidad a } \\
\text { las formas nativas de asparaginasa }\end{array}$ \\
\hline Asparaginasa de $E$. coli nativa & $1.2 \pm 0.3$ & $14-23$ & Tratamiento de primera línea en la mayoría de los protocolos \\
\hline $\begin{array}{l}\text { Asparaginasa de } \\
\text { E. chrysanthemi }\end{array}$ & $0.6 \pm 0.1$ & $7-15$ & $\begin{array}{l}\text { Tratamiento de segunda o tercera línea en casos de hipersensibilidad a } \\
\text { las formas derivadas de asparaginasa de E. coli nativa }\end{array}$ \\
\hline
\end{tabular}

Tabla 2. Fármacos utilizados frecuentemente en niños con enfermedades hematooncológicas asociadas con pancreatitis aguda

\begin{tabular}{|l|l|}
\hline Grupo & Fármaco \\
\hline Quimioterapéuticos & $\begin{array}{l}\text { L-asparaginasa } \\
\text { Mercaptopurina } \\
\text { Citarabina } \\
\text { Ifosfamida } \\
\text { Cisplatino } \\
\text { Doxorubicina } \\
\text { Metotrexato }\end{array}$ \\
\hline Corticosteroides & $\begin{array}{l}\text { Dexametasona } \\
\text { Prednisona }\end{array}$ \\
\hline Inmunosupresores & $\begin{array}{l}\text { Tacrolimus } \\
\text { Ciclosporina }\end{array}$ \\
\hline Antibióticos & $\begin{array}{l}\text { Trimetoprima-sulfametoxazol } \\
\text { Eritromicina }\end{array}$ \\
\hline
\end{tabular}

\section{Trasplante de células progenitoras hematopoyéticas}

El TCPH es un procedimiento cada día más asequibley empleado en el tratamiento de enfermedades hematooncológicas y de inmunodeficiencias. La PA se describe en el 3.5\% de los niños después de un TCPH. La etiología es multifactorial y se atribuye a factores involucrados en el procedimiento del trasplante ${ }^{20}$. Un estudio que correlacionó variables clínicas y hallazgos en autopsias de pacientes con antecedente de TCPH determinó una prevalencia de pancreatitis del $28 \%$. En el análisis multivariado se encontraron los siguientes factores de riesgo: enfermedad del injerto contra el huésped, afección hepática, infecciones y mayor estancia hospitalaria ${ }^{21}$.

Otros factores que deben considerarse en los niños con PA y TCPH son los fármacos inmunosupresores ${ }^{22}$ utilizados antes y después del trasplante, así como las infecciones concomitantes por adenovirus o citomegalovirus ${ }^{23,24}$.

\section{Evaluación y diagnóstico}

\section{Clínica}

La PA en los niños con cáncer debe sospecharse ante la presencia de alguno de los factores previamente descritos. Los pacientes inician con dolor abdominal epigástrico, náusea y vómito. Los lactantes presentan menos síntomas, por lo que para su diagnóstico se requiere un alto índice de sospecha ${ }^{25}$.

\section{Estudios de laboratorio}

Los marcadores bioquímicos disponibles para el diagnóstico de PA son la lipasa y la amilasa séricas. La lipasa generalmente aumenta dentro de las primeras 6 horas del inicio de los síntomas; las concentraciones séricas alcanzan su valor máximo en 24 a 30 horas y pueden permanecer elevadas por más de 1 semana. La amilasa aumenta más rápido que la lipasa y con frecuencia puede normalizarse 24 horas después del inicio de los síntomas, lo que limita su utilidad en pacientes con una presentación tardía ${ }^{2}$. A medida que los riñones excretan amilasa y lipasa pueden observarse elevaciones no pancreáticas de estas enzimas en pacientes con lesión o enfermedad renal ${ }^{26}$.

Otras pruebas de laboratorio que se sugiere monitorear son la citometría hemática, los electrolitos séricos, las concentraciones de calcio, los marcadores de la función hepática, el nitrógeno ureico, la creatinina y los triglicéridos.

\section{Estudios de imagen}

No se recomienda solicitar estudios de imagen en la fase inicial, principalmente cuando se tiene alta sospecha clínica de PA y se documenta una elevación de los marcadores bioquímicos. La utilidad de los estudios de 
imagen radica en detectar complicaciones de la $\mathrm{PA}^{2}$. La ecografía tiene un excelente perfil de seguridad, aunque la evaluación del páncreas puede estar limitada debido a estructuras interferentes, como gas intestinal y tejido adiposo en los niños con obesidad. A pesar de la poca sensibilidad ${ }^{27}$ es útil, en especial cuando se sospecha pancreatitis biliar ${ }^{28}$.

El criterio estándar para el diagnóstico por imagen es aún la tomografía computarizada con contraste. Debe solicitarse cuando los marcadores séricos se encuentren en valores bajos o normales, o se sospechen complicaciones. El contraste intravenoso es necesario para distinguir áreas de necrosis en el páncreas ${ }^{28}$. Lo ideal es solicitar la tomografía computarizada al menos 96 horas después del inicio de los síntomas ${ }^{29}$. El índice de gravedad de la tomografía computarizada (puntuación de Balthazar) en pacientes pediátricos proporciona una sensibilidad del $81 \%$, una especificidad del $76 \%$, un valor predictivo positivo del $62 \%$ y un valor predictivo negativo del $90 \%{ }^{30}$.

Las imágenes de resonancia magnética y la colangiopancreatografía por resonancia magnética no se utilizan como técnicas de imagen inicial en la $\mathrm{PA}^{2}$.

\section{Diagnóstico diferencial}

El dolor abdominal en los niños con cáncer es un síntoma frecuente, por lo que el abordaje y el diagnóstico son un reto clínico. Los pacientes con neutropenia (neutrófilos < 500/ $\mu$ l) presentan un mayor riesgo de desarrollar enterocolitis neutropénica ${ }^{31}$. Los síntomas más comunes de la enterocolitis neutropénica son dolor abdominal, diarrea y fiebre. Esta tríada de síntomas se encuentra hasta en el $78 \%$ de los pacientes; otros síntomas comunes son náusea, vómito y distensión abdominal ${ }^{32}$.

La apendicitis aguda acurre en el $1 \%$ de los niños con LLA y puede desarrollarse durante la inducción a la remisión ${ }^{33}$. El estreñimiento es una causa de dolor abdominal y se ha observado con mayor frecuencia en niños después de haber recibido quimioterapia con vincristina ${ }^{34}$.

\section{Tratamiento}

\section{Fluidoterapia}

La terapia con líquidos intravenosos es el pilar fundamental en el tratamiento de la PA en los niños. La reanimación con líquidos mantiene un adecuado volumen circulante y un correcto flujo urinario ${ }^{35}$. Además, se describe que la correcta terapia hídrica previene posibles complicaciones. Se cree que la patogenia de la PA y la progresión a formas graves son secundarias a alteraciones en la microcirculación del páncreas por eventos que incluyen hipovolemia, aumento de la permeabilidad capilar y formación de microtrombos ${ }^{36}$. Es importante recordar que los niños con cáncer presentan mayor riesgo de trombosis ${ }^{37}$.

No existe consenso en cuanto al tipo de soluciones y la cantidad que se debe utilizar en la reanimación inicial en los niños con PA. Sin embargo, se ha demostrado que el manejo agresivo de fluidos cristaloides durante las primeras 24 horas es bien tolerado y mejora la evolución clínica y el pronóstico. Si hay compromiso del estado hemodinámico, se recomienda iniciar con una carga de cristaloides de 10-20 ml $/ \mathrm{kg}$. Posteriormente, los líquidos intravenosos de mantenimiento se deben continuar con 1.5-2 veces los requerimientos basales, y monitorizar la producción de orina durante las siguientes 24-48 horas².

\section{Analgesia}

El dolor abdominal epigástrico es el síntoma más frecuente de la PA en los pacientes pediátricos ${ }^{38}$. El control del dolor es un objetivo terapéutico importante en la PA y generalmente implica el uso de analgésicos de acción central y periférica. La morfina intravenosa, u otro opiáceo, se debe utilizar para el dolor generado por la PA que no responde a la administración de paracetamol ni de analgésicos antinflamatorios no esteroideos. Los opiáceos pueden ser una opción adecuada para el tratamiento del dolor relacionado con la PA y pueden disminuir la necesidad de analgesia complementaria ${ }^{39}$. Si en el centro hospitalario se dispone, se puede consultar al departamento de algología para casos de dolor intenso u optimizar el tratamiento analgésico.

\section{Alimentación y nutrición}

Tradicionalmente, el tratamiento de la PA incluía ayuno y el inicio temprano de la nutrición parenteral total (NPT). En la actualidad se recomienda que los niños con PA leve inicien la alimentación por vía oral o la nutrición enteral de forma temprana (dentro de las primeras 48-72 horas de la presentación). Esto disminuye el riesgo de disfunción orgánica y el tiempo de estancia hospitalaria ${ }^{40}$.

La NPT proporciona el requerimiento calórico y los nutrimentos necesarios para compensar el estado 
catabólico que existe en los niños con PA. La NPT debe considerarse cuando la nutrición enteral no es posible durante un periodo prolongado (más de 5-7 días), como en caso de íleo, fístulas complejas o síndrome compartimental abdominal ${ }^{2}$. No deben restringirse las proteínas, la glucosa ni los lípidos por vía parenteral; sin embargo, se debe monitorear la glucosa, ya que puede haber una alteración en la secreción de insulina secundaria a la inflamación pancreática ${ }^{41}$.

\section{Antibióticos}

Los antibióticos no deben utilizarse sistemáticamente en el tratamiento de la PA, excepto en caso de necrosis infectada documentada y en niños hospitalizados con pancreatitis necrótica que no mejoran clínicamente. Los antibióticos que penetran en el tejido necrótico (el imipenem y las cefalosporinas de tercera generación) pueden retrasar la intervención quirúrgica y disminuir la morbilidad y la mortalidad ${ }^{41,42}$. En los niños con enfermedades hematooncológicas deben considerarse otros factores para la administración de antibióticos, como la presencia de neutropenia y el uso de fármacos mielosupresores.

\section{Otros}

La octreotida inhibe las secreciones de diversos órganos endocrinos, incluyendo las enzimas digestivas pancreáticas, por lo que su uso podría disminuir la inflamación del páncreas ${ }^{43}$. Algunos autores sugieren que la octreotida es útil para prevenir la pancreatitis en la reintroducción de L-asparaginasa ${ }^{44}$. Sin embargo, son necesarios más estudios que demuestren la utilidad real, los efectos adversos, las dosis y el tiempo de administración de la terapia profiláctica con octreotida.

En población pediátrica no se recomienda el uso de antioxidantes, inhibidores de proteasas ni probióticos en el tratamiento de la $\mathrm{PA}^{2}$.

\section{Pronóstico}

El pronóstico de la PA en los niños es favorable en comparación con los adultos. La mayor mortalidad se asocia con una enfermedad sistémica como el cáncer, pero en general es baja e inferior al $5 \%$ en la mayoría de las cohortes, incluso con ingresos a la unidad de cuidados intensivos ${ }^{45}$. Una revisión sistemática que analizó diversos factores para el desarrollo de PA por
L-asparaginasa informó que la pancreatitis grave puede presentarse en el $7-66 \%$ de los niños ${ }^{46}$. La limitante en este estudio fue que se utilizaron los criterios de Atlanta de 2012 para definir la pancreatitis grave ${ }^{47}$, y esta clasificación no fue desarrollada para su uso en niños.

En la PA leve, los síntomas suelen resolverse en la primera semana y no se deben solicitar sistemáticamente determinaciones de la amilasa y la lipasa séricas, a menos que el paciente continúe con signos y síntomas de inflamación pancreática. El propósito de monitorear las enzimas pancreáticas no es vigilar el estado del páncreas, sino detectar complicaciones. Sin embargo, los estudios de son más sensibles que la amilasa y la lipasa para diagnosticar complicaciones de la $P A^{48}$.

\section{Complicaciones}

Las complicaciones de inicio temprano en la PA incluyen la disfunción multiorgánica y el estado de choque. La acumulación de líquido peripancreático se observa en la fase aguda de la pancreatitis y tiende a resolverse de manera espontánea. Los pseudoquistes a menudo son asintomáticos y suelen tratarse de forma conservadora; se asocian con dolor abdominal, vómito y fiebre, y llegan a infectarse en el $10-15 \%$ de los casos $^{49}$.

Entre las complicaciones crónicas asociadas con PA en los niños con leucemia se han descrito la pancreatitis crónica y la diabetes mellitus dependiente de insulina ${ }^{50}$.

La recurrencia de PA por L-asparaginasa se presenta en el $7-62.5 \%$ de los casos después de un primer evento ${ }^{51}$. No existe consenso sobre la reintroducción de la L-asparaginasa, por lo que se recomienda que se administre 48 horas después de no presentar síntomas de PA, cuando los valores de amilasa y de lipasa se encuentran debajo de tres veces el valor normal, y no aparecen pseudoquistes o necrosis en las imágenes. Si los pacientes presentan un nuevo episodio de PA, se sugiere suspender el tratamiento con L-asparaginasa ${ }^{52}$.

Los niños en tratamiento por alguna enfermedad hematooncológica son más susceptibles a desarrollar PA, enfermedad que debe sospecharse ante la presencia de dolor abdominal, náusea y vómito. Una edad mayor, el tipo de L-asparaginasa y múltiples dosis parecen conferir un mayor riesgo de presentar PA. El inicio temprano de la vía oral, la adecuada terapia hídrica y una óptima analgesia son las medidas terapéuticas que mejoran la inflamación pancreática y pueden 
prevenir complicaciones. Los estudios de imagen deben limitarse y se solicitarán cuando la etiología de la PA no sea clara o se sospechen complicaciones. Futuros estudios definirán el papel de fármacos como la octreotida en la prevención de la pancreatitis de repetición asociada con L-asparaginasa.

\section{Responsabilidades éticas}

Protección de personas y animales. Los autores declaran que para esta investigación no se han realizado experimentos en seres humanos ni en animales.

Confidencialidad de los datos. Los autores declaran que han seguido los protocolos de su centro de trabajo sobre la publicación de datos de pacientes.

Derecho a la privacidad y consentimiento informado. Los autores declaran que en este artículo no aparecen datos de pacientes.

\section{Conflicto de intereses}

Los autores declaran no tener ningún conflicto de intereses.

\section{Financiamiento}

Ninguno.

\section{Bibliografía}

1. Stefanović M, Jazbec J, Lindgren F, Bulajić M, Löhr M. Acute pancreatitis as a complication of childhood cancer treatment. Cancer Med. 2016;5:827-36.

2. Abu-El-Haija M, Kumar S, Quiros JA, Balakrishnan K, Barth B, Bitton S, et al. Management of acute pancreatitis in the pediatric population: a clinical report from the North American Society for Pediatric Gastroenterology, Hepatology and Nutrition Pancreas Committee. J Pediatr Gastroenterol Nutr. 2018;66:159-76.

3. Morinville VD, Barmada MM, Lowe ME. Increasing incidence of acute pancreatitis at an American pediatric tertiary care center: is greater awareness among physicians responsible? Pancreas. 2010;39:5-8.

4. Treepongkaruna S, Thongpak N, Pakakasama S, Pienvichit P, Sirachainan $\mathrm{N}$, Hongeng $\mathrm{S}$. Acute pancreatitis in children with acute lymphoblastic leukemia after chemotherapy. J Pediatr Hematol Oncol. 2009;31:812-5.

5. Raja RA, Schmiegelow K, Albertsen BK, Prunsild K, Zeller B, Vaitkeviciene $\mathrm{G}$, et al. Asparaginase-associated pancreatitis in children with acute lymphoblastic leukaemia in the NOPHO ALL2008 protocol. $\mathrm{Br} J$ Haematol. 2014;165:126-33.

6. García-Dávila M, Villalpando-Carrión S, Heller-Rouassant S, Solís-Perales NE, Ortiz-Ramírez NE, López-Contreras N. Factores asociados al desarrollo de complicaciones en pancreatitis aguda en niños. Bol Med Hosp Infant Mex. 2009;66:160-6.

7. Ridaura-Sanz C, Arias-Lima A. Pancreatitis aguda en niños. Una perspectiva desde la autopsia. Acta Pediatr Mex. 2019;40:11-5.

8. Majbar AA, Cusick E, Johnson P, Lynn RM, Hunt LP, Shield JP. Incidence and clinical associations of childhood acute pancreatitis. Pediatrics. 2016;138:1-7.

9. Suzuki M, Sai JK, Shimizu T. Acute pancreatitis in children and adolescents. World J Gastrointest Pathophysiol. 2014:5:416-26.

10. Vargas-Quevedo E, Ordóñez-Gutiérrez E, Trejo-Gómora JE, Chávez-Aguilar LA, Peña-Vélez R. "Seronegative" catastrophic antiphospholipid syndrome in pediatrics: clinical case. Rev Chil Pediatr. 2018;89:236-40.
11. Pérez-Cuevas R, Muñoz-Hernández 0 . Importancia de la salud pública dirigida a la niñez y la adolescencia en México. Bol Med Hosp Infant Mex. 2014;71:126-33.

12. Liu C, Yang W, Devidas M, Cheng C, Pei D, Smithet C, et al. Clinical and genetic risk factors for acute pancreatitis in patients with acute lymphoblastic leukemia. J Clin Oncol. 2016;34:2133-40.

13. Ono $Y$, Kimura T, Nakano I, Furukawa M, Ito T, Sakamoto S, et al. Acute pancreatitis induced by hypercalcemia associated with adult T-ceII leukaemia: a case report. J Gastroenterol Hepatol. 1996;11:193-5.

14. Ridola V, Buonuomo PS, Maurizi P, Putzulu R, Annunziata ML, Pietrini D, et al. Severe acute hypertriglyceridemia during acute lymphoblastic leukemia induction successfully treated with plasmapheresis. Pediatr Blood Cancer. 2008;50:378-80.

15. Moscardó Guilleme C, Fernández Delgado R, Sevilla Navarro J, Astigarraga Aguirre I, Rives Solà S, Sánchez de Toledo Codina J, et al. Actualización del tratamiento con L-asparaginasa en pediatría. An Pediatr (Barc). 2013;79:329.e1-11.

16. Flores-Calderón J, Exiga-González E, Morán-Villota S, Martín-Trejo J, Yamamoto-Nagano A. Acute pancreatitis in children with acute lymphoblastic leukemia treated with L-asparaginase. J Pediatr Hematol Oncol. 2009;31:790-3.

17. Knoderer HM, Robarge J, Flockhart DA. Predicting asparaginase-associated pancreatitis. Pediatr Blood Cancer. 2007;49:634-9.

18. Inaba $\mathrm{H}$, Pui $\mathrm{CH}$. Glucocorticoid use in acute lymphoblastic leukaemia. Lancet Oncol. 2010;11:1096-106.

19. Teuffel O, Kuster SP, Hunger SP, Conter V, Hitzler J, Ethier MC, et al. Dexamethasone versus prednisone for induction therapy in childhood acute lymphoblastic leukemia: a systematic review and meta-analysis. Leukemia. 2011;25:1232-8.

20. Werlin SL, Casper J, Antonson D, Calabro C. Pancreatitis associated with bone marrow transplantation in children. Bone Marrow Transplant. 1992;10:65-9.

21. Ko CW, Gooley T, Schoch HG, Myerson D, Hackman RC, Shulman HM, et al. Acute pancreatitis in marrow transplant patients: prevalence at autopsy and risk factor analysis. Bone Marrow Transplant. 1997;20: 1081-6.

22. Badalov N, Baradarian R, Iswara K, Li J, Steinberg W, Tenner S. Drug-induced acute pancreatitis: an evidence-based review. Clin Gastroenterol Hepatol. 2007;5:648-61.

23. Niemann TH, Trigg ME, Winick N, Penick GD. Disseminated adenoviral infection presenting as acute pancreatitis. Hum Pathol. 1993;24:1145-8.

24. Tomonari A, Takahashi S, Takasugi K, Ooi J, Tsukada N, Konuma T, et al. Pancreatic hyperamylasemia and hyperlipasemia in association with cytomegalovirus infection following unrelated cord blood transplantation for acute myelogenous leukemia. Int J Hematol. 2006;84:438-40.

25. Park AJ, Latif SU, Ahmad MU, Bultron G, Orabi Al, Bhandari V, et al. A comparison of presentation and management trends in acute pancreatitis between infants/toddlers and older children. J Pediatr Gastroenterol Nutr. 2010;51:167-70.

26. Lippi G, Valentino M, Cervellin G. Laboratory diagnosis of acute pancreatitis: in search of the Holy Grail. Crit Rev Clin Lab Sci. 2012;49:18-31.

27. Tenner S, Baillie J, DeWitt J, Vege SS, American College of Gastroenterology. American College of Gastroenterology guideline: management of acute pancreatitis. Am J Gastroenterol. 2013;108:1400-15.

28. Baker ME, Nelson RC, Rosen MP, Blake MA, Cash BD, Hindman NM, et al. ACR Appropriateness Criteria $\AA^{\circledR}$ acute pancreatitis. Ultrasound $\mathrm{Q}$. 2014:30:267-73

29. Zhao K, Adam SZ, Keswani RN, Horowitz JM, Miller FH. Acute pancreatitis: revised Atlanta Classification and the role of cross-sectional imaging. AJR Am J Roentgenol. 2015;205:W32-41.

30. Lautz TB, Turkel G, Radhakrishnan J, Wyers M, Chin AC. Utility of the computed tomography severity index (Balthazar score) in children with acute pancreatitis. J Pediatr Surg. 2012;47:1185-91.

31. Aksoy DY, Tanriover MD, Uzun O, Zarakolu P, Ercis S, Ergüven S, et al. Diarrhea in neutropenic patients: a prospective cohort study with emphasis on neutropenic enterocolitis. Ann Oncol. 2007;18:183-9.

32. Rodrigues FG, Dasilva G, Wexner SD. Neutropenic enterocolitis. World J Gastroenterol. 2017;23:42-7.

33. Perrino M, Fernandez-Pineda I, Zhou Y, Sandoval J, Jeha S, Rubnitz JE, et al. Acute appendicitis in children with leukemia: unique diagnostic process, management, and outcome. Blood. 2015;126:4872.

34. Pashankar FD, Season JH, McNamara J, Pashankar DS. Acute constipation in children receiving chemotherapy for cancer. J Pediatr Hematol Oncol. 2011;33:e300-3.

35. Aggarwal A, Manrai M, Kochhar R. Fluid resuscitation in acute pancreatitis. World J Gastroenterol. 2014;20:18092-103.

36. Gardner TB, Vege SS, Pearson RK, Chari ST. Fluid resuscitation in acute pancreatitis. Clin Gastroenterol Hepatol. 2008:6:1070-6.

37. Ko RH, Thornburg CD. Venous thromboembolism in children with cancer and blood disorders. Front Pediatr. 2017;5:1-7.

38. Bai HX, Lowe ME, Husain SZ. What have we learned about acute pancreatitis in children? J Pediatr Gastroenterol Nutr. 2011;52:262-70.

39. Basurto Ona X, Rigau Comas D, Urrutia G. Opioids for acute pancreatitis pain. Cochrane Database Syst Rev. 2013;(7):CD009179. 
40. Fan BG, Andren-Sandberg A. Acute pancreatitis. N Am J Med Sci. 2010;2:211-4.

41. Working Group IAP/APA Acute Pancreatitis Guidelines. IAP/APA evidence-based guidelines for the management of acute pancreatitis. Pancreatology. 2013;13:e1-15.

42. Villatoro E, Mulla M, Larvin M. Antibiotic therapy for prophylaxis against infection of pancreatic necrosis in acute pancreatitis. Cochrane Database Syst Rev. 2010;(5):CD002941.

43. Wu SF, Chen AC, Peng CT, Wu KH. Octreotide therapy in asparaginase-associated pancreatitis in childhood acute lymphoblastic leukemia Pediatr Blood Cancer. 2008;51:824-5.

44. Tokimasa S, Yamato K. Does octreotide prevent L-asparaginase-associated pancreatitis in children with acute lymphoblastic leukaemia? $\mathrm{Br}$ Haematol. 2012;157:381-2.

45. Goday PS, Wakeham M, Kuhn EM, Collins MM, Werlin SL. Acute pancreatitis in the pediatric intensive care unit. J Pediatr Gastroenterol Nutr. 2015;61:108-12.

46. Oparaji JA, Rose F, Okafor D, Howard A, Turner RL, Orabi Al, et al. Risk factors for asparaginase-associated pancreatitis: a systematic review. J Clin Gastroenterol. 2017;51:907-13.
47. Banks PA, Bollen TL, Dervenis C, Gooszen HG, Johnson CD, Sarr MG et al. Classification of acute pancreatitis - 2012: revision of the Atlanta classification and definitions by international consensus. Gut. 2013;62:102-11.

48. Banks PA, Freeman ML; Practice Parameters Committee of the American College of Gastroenterology. Practice guidelines in acute pancreatitis. Am J Gastroenterol. 2006:101:2379-400.

49. Boerma D, van Gulik TM, Obertop H, Gouma DJ. Internal drainage of infected pancreatic pseudocysts: safe or sorry? Dig Surg. 1999;16:501-5

50. Wolthers BO, Mogensen PR, Frandsen TL, Abrahamsson J, Behrendtz M, Heyman M, et al. Insulin-dependent diabetes: a chronic complication to acute pancreatitis in childhood acute lymphoblastic leukemia. Pediatr Blood Cancer. 2019;66:1-4.

51. Kearney SL, Dahlberg SE, Levy DE, Voss SD, Sallan SE, Silverman LB. Clinical course and outcome in children with acute lymphoblastic leukemia and asparaginase-associated pancreatitis. Pediatr Blood Cancer. 2009;53:162-7.

52. Raja RA, Schmiegelow K, Frandsen TL. Asparaginase-associated pancreatitis in children. Br J Haematol. 2012;159:18-27. 